\title{
Prevalência de pacientes com câncer avançado atendidos num serviço de cuidados paliativos no Estado de Alagoas
}

\author{
Prevalence of patients with advanced cancer treated at a palliative care service in the \\ State of Alagoas
}

\section{Prevalencia de pacientes con cáncer avanzado atendidos en un servicio de cuidados paliativos del Estado de Alagoas}

Weslley Paixão da Silva ${ }^{1 *}$, Carolina Záu Serpa de Araujo², Laís Záu Serpa de Araujo.

\begin{abstract}
RESUMO
Objetivo: Identificar a prevalência de pacientes com câncer avançado atendidos num serviço de Cuidados Paliativos. Métodos: Estudo quantitativo, descritivo, retrospectivo e prospectivo, através dos prontuários e contato telefônico com familiares de pacientes atendidos no serviço de cuidados paliativos. Resultados: Dos 770 prontuários, 58,31\% eram de mulheres, a média da idade na admissão foi 61,92. Os tumores de cabeça e pescoço, pulmão, colo de útero, mama e próstata foram os mais prevalentes. Dor, constipação e dispneia foram os sintomas mais encontrados. Identificou-se que $23,64 \%$ dos pacientes não foram submetidos a nenhum tipo de tratamento modificador da doença antes de serem encaminhados ao serviço de Cuidados Paliativos. Dos pacientes atendidos, $92,21 \%$ não realizaram tratamento modificador da doença após o encaminhamento ao serviço. Conclusão: Os pacientes que recorreram à assistência paliativa são predominantemente mulheres, de idades mais avançadas, que necessitavam de manejo apropriado dos sintomas. Pode-se aferir que há relação entre os tipos de cânceres mais prevalentes e o sexo feminino ser mais predominante no serviço de Cuidados Paliativos.
\end{abstract}

Palavras-chaves: Cuidados paliativos, Doente terminal, Oncologia, Câncer, Assistência terminal.

\begin{abstract}
Objective: To identify the prevalence of service of patients with advanced cancer treated with numerical palliative care. Methods: Quantitative, descriptive, retrospective and prospective study, through medical records and telephone contact with family members of patients treated at the palliative care service. Results: Of the 770 medical records, $58.31 \%$ were women, the average age at admission was 61.92. Tumors of the head and neck, lung, cervix, breast and prostate were the most prevalent. Pain, constipation and dyspnea were the most common symptoms. It was identified that $23.64 \%$ of the patients did not undergo any type of disease-modifying treatment before being referred to the Palliative Care service. Of the patients seen, $92.21 \%$ did not undergo disease-modifying treatment after being referred to the service. Conclusion: Patients who resorted to palliative care are predominantly women, of older ages, who needed appropriate management of symptoms. It can be verified that there is a relationship between the most prevalent types of cancers and the female sex is more prevalent in the Palliative Care service.
\end{abstract}

Keywords: Palliative care, Terminally III, Medical oncology, Cancer, Terminal care.

\section{RESUMEN}

Objetivo: Identificar la prevalencia de atención de pacientes con cáncer avanzado tratados con cuidados paliativos numéricos. Métodos: Estudio cuantitativo, descriptivo, retrospectivo y prospectivo, mediante historia clínica y contacto telefónico con familiares de pacientes atendidos en el servicio de cuidados paliativos. Resultados: De las 770 historias clínicas, el 58,31\% eran mujeres, la edad promedio al ingreso fue de 61,92 años. Los tumores de cabeza y cuello, pulmón, cuello uterino, mama y próstata fueron los más prevalentes. El dolor, el estreñimiento y la disnea fueron los síntomas más frecuentes. Se identificó que el $23,64 \%$ de los pacientes no se sometió a ningún tipo de tratamiento modificador de la enfermedad antes de ser derivados al servicio de Cuidados Paliativos. De los pacientes atendidos, el 92,21\% no recibió tratamiento modificador de la enfermedad tras su derivación al servicio. Conclusión: Los pacientes que acudieron a cuidados paliativos son predominantemente mujeres, de mayor edad, que precisaron un adecuado manejo de la sintomatología. Se puede verificar que existe una relación entre los tipos de cáncer más prevalentes y el sexo femenino es más prevalente en el servicio de Cuidados Paliativos.

Palabra chave: Cuidados paliativos, Enfermo terminal, Oncologia médica, Cáncer, Cuidado terminal.

1 Universidade Estadual de Ciências da Saúde de Alagoas (UNCISAL), Maceió - AL.

*E-mail: weslleywps.med@gmail.com

2 Hospital Santa Casa de Misericórdia de Maceió, Maceió - AL.

Fontes de Financiamento: Fundação de Amparo à Pesquisa do Estado de Alagoas. Bolsa de Iniciação Científica. Processo: 60030 000819/2017 (16). Direcionado a Weslley Paixão da Silva 


\section{INTRODUÇÃO}

De acordo com o Instituto Nacional do Câncer (INCA) são previstos 625 mil novos diagnósticos de neoplasias durante o triênio 2020-2022 no país, o que, sem dúvida, exigirá novos olhares e propostas diferenciadas para os portadores de câncer avançado (INCA, 2019). Com a finalidade de somar esforços nessa luta, no Brasil, a Lei oํ 12.732/2012 estabeleceu que todo portador de neoplasia maligna tem direito a ser assistido no Sistema Único de Saúde (SUS) no prazo de até 60 dias, ou em prazo menor, caso tenha necessidade médica (BRASIL, 2012).

Sabe-se, também, que a prevenção e o diagnóstico precoce não têm sido eficazes, o que resulta sobretudo na descoberta tardia e num estágio avançado do câncer, muitas vezes, sem nenhum tratamento prévio e modificador da doença (FURTADO MEMF e LEITE DMC, 2017; VISENTIN A, et al., 2018). Outrossim, a assistência oncológica pressupõe na sua complexidade a adoção de um modelo de atenção e de conforto que amenize o sofrimento humano ante a ausência de possibilidades curativas, sendo, em última análise, o doente o principal beneficiado em observância ao respeito à dignidade humana (VISENTIN A, et al., 2018; OLIVEIRA LC, 2019).

Nessa perspectiva, a instituição e ampliação dos serviços especializados em Cuidados Paliativos (CP) constituem meios eficazes de auxílio ao indivíduo em adoecimento, uma vez que a Organização Mundial de Saúde (OMS) defende este suporte como fundamental para que as diversas áreas de saúde proporcionem qualidade de vida e superação das repercussões negativas advindas do enfrentamento da doença (OMS, 2002). Ribeiro JR e Poles K (2019) afirmam que "os cuidados curativos e paliativos não deveriam ser considerados como conceitos excludentes, mas, sim, complementares".

Segundo Oliveira MBP, et al. (2017) a abordagem paliativa é necessária para o controle dos sintomas provenientes de doenças em estágios avançados. Em concordância a isso, Kavalieratos D, et al. (2016) e Haun MW, et al. (2017) afirmam em seus estudos que quanto mais precocemente forem inseridas as condutas paliativas, mais perceptíveis são os benefícios para a qualidade de vida e a diminuição da intensidade dos sintomas apresentados.

Diante do exposto, justifica-se este trabalho, pela importância do tema e pretendendo subsidiar a tomada de decisão nas políticas públicas. O objetivo deste estudo foi de identificar a prevalência de pacientes com câncer avançado atendidos num serviço de Cuidados Paliativos no Estado de Alagoas.

\section{MÉTODOS}

O projeto de pesquisa que gerou este trabalho foi elaborado de acordo com as recomendações éticas contidas na resolução 466/2012, do Conselho Nacional de Saúde. Foi submetido e aprovado por um comitê de ética em pesquisa e recebeu o número CAAE 67285917.4.0000.0039.

O método foi quantitativo, descritivo, retrospectivo e prospectivo. Utilizou-se como estratégia para coleta de dados a verificação direta nos prontuários dos pacientes e planilhas ambulatoriais e hospitalares do serviço. Quando identificado que o paciente perdera o seguimento, os pesquisadores entraram em contato com um familiar, cujo número de telefone constava no prontuário, para identificar a razão da perda do seguimento.

A pesquisa foi desenvolvida em um serviço de cuidados paliativos oncológicos de um hospital terciário situado no Estado de Alagoas. Este atende exclusivamente pacientes do Sistema Único de Saúde, portadores de câncer, encaminhados em função do agravamento da doença, do seu estado e da inexistência de possibilidades terapêuticas curativas.

A amostra foi composta por prontuários de pacientes que foram atendidos no serviço de Cuidados Paliativos Oncológicos e foram a óbito no período de 13 de agosto de 2013 até 13 de agosto de 2017. Este período foi escolhido por representar a data inicial de funcionamento, 13 de agosto de 2013, até completar 4 anos no dia 13 de agosto de 2017. Os critérios de inclusão foram todos os prontuários de pacientes atendidos no e que foram a óbito e foram excluídos os prontuários dos pacientes que continuavam sendo acompanhados. 
A variável primária foi o tipo de câncer e as variáveis secundárias foram idade na admissão; idade no óbito; origem do encaminhamento; sintomas prevalentes, performance na admissão, tratamentos antes e após a admissão nos cuidados paliativos, internações e os dados complementares foram idade; sexo; naturalidade; local de domicílio e do óbito. As informações coletadas foram armazenadas em planilha eletrônica e os dados foram estudados através de análise descritiva.

\section{RESULTADOS}

Neste estudo foram incluídos 770 prontuários de pacientes oncológicos, admitidos no serviço de Cuidados Paliativos, sendo $58,31 \%$ do sexo feminino e $41,69 \%$ do sexo masculino. Da amostra estudada, a faixa etária variou de 20 a 97 anos, média de 61,92, mediana de 62 e a moda 59. Desses, 4,81\% tinham idade no momento da admissão entre 20 a 35 anos; $27,01 \%$ de 36 a 55; 47,53\% de 56 a 75 e 20,65\% de 76 a 97 anos. Quanto à cidade de domicílio dos pacientes, $58,70 \%$ foram provenientes de uma cidade do Estado de Alagoas. A informação sobre a naturalidade não foi registrada em $71,69 \%$ dos prontuários e $24,03 \%$ estavam registrados como naturais de Alagoas.

Identificou-se que $86,23 \%$ dos doentes foram encaminhados pelo Centro de Oncologia do próprio hospital que mantém o serviço de Cuidados Paliativos, 5,32\% pelo Hospital 1 que é um complexo hospitalar especializado em urgência e emergência, 2,60\% pela Radioterapia e 1,95\% pela Cirurgia Oncológica. Verificou-se que $73,64 \%$ dos pacientes foram a óbito no próprio serviço de Cuidados Paliativos. A faixa etária no óbito variou de 20 a 97 anos, média de 61,02, mediana de 61 e distribuição bimodal 59 e 65. Do total de prontuários levantados, $25,58 \%$ dos pacientes perderam seguimento (Tabela 1).

Tabela 1 - Características epidemiológicas dos pacientes atendidos no Serviço de Cuidados Paliativos

\begin{tabular}{|c|c|c|}
\hline Variáveis & $\mathbf{N}$ & $\%$ \\
\hline \multicolumn{3}{|l|}{ Sexo } \\
\hline Masculino & 321 & $41,69 \%$ \\
\hline Feminino & 449 & $58,31 \%$ \\
\hline \multicolumn{3}{|l|}{ Idade na admissão } \\
\hline $20-35$ & 37 & $4,81 \%$ \\
\hline $36-55$ & 208 & $27,01 \%$ \\
\hline $56-75$ & 366 & $47,53 \%$ \\
\hline $76-97$ & 159 & $20,65 \%$ \\
\hline \multicolumn{3}{|l|}{ Cidade de domicílio } \\
\hline Maceió-AL & 452 & $58,70 \%$ \\
\hline Outras & 318 & $41,30 \%$ \\
\hline \multicolumn{3}{|l|}{ Naturalidade } \\
\hline Alagoas & 185 & $24,03 \%$ \\
\hline Outros & 33 & $4,28 \%$ \\
\hline Dados não obtidos & 552 & $71,69 \%$ \\
\hline \multicolumn{3}{|l|}{ Encaminhamento } \\
\hline Centro de Oncologia & 664 & $86,23 \%$ \\
\hline Cirurgia Oncológica & 15 & $1,95 \%$ \\
\hline Hospital 1 & 41 & $5,32 \%$ \\
\hline Radioterapia & 20 & $2,60 \%$ \\
\hline Outros & 30 & $3,90 \%$ \\
\hline \multicolumn{3}{|l|}{ Seguimento } \\
\hline Pacientes que perderam seguimento & 197 & $25,58 \%$ \\
\hline \multicolumn{3}{|l|}{ Idade no óbito } \\
\hline $20-35$ & 28 & $3,63 \%$ \\
\hline $36-55$ & 165 & $21,42 \%$ \\
\hline $56-75$ & 273 & $35,45 \%$ \\
\hline $76-97$ & 106 & $13,76 \%$ \\
\hline \multicolumn{3}{|l|}{ Local do óbito } \\
\hline Serviço de Cuidados Paliativos Oncológicos & 567 & $73,64 \%$ \\
\hline
\end{tabular}


Em relação à distribuição dos tipos de câncer mais prevalentes no serviço, constatou-se que $15,32 \%$ foram tumores de Cabeça e Pescoço, 13,77\% de Pulmão, 12,86\% de Colo de útero, 10,65\% de Mama e 6,75\% de Próstata (Tabela 2).

Tabela 2 - Tipos de câncer dos pacientes atendidos no Serviço de Cuidados Paliativos.

\begin{tabular}{lcc}
\hline Sítio Primário da Doença & N & $\%$ \\
\hline Cabeça e Pescoço & 118 & 15,32 \\
Pulmão & 106 & 13,77 \\
Colo de Útero & 99 & 12,86 \\
Mama & 82 & 10,65 \\
Próstata & 52 & 6,75 \\
Desconhecido & 42 & 5,45 \\
Estômago & 40 & 5,19 \\
Colón e Reto & 37 & 4,81 \\
Esôfago & 29 & 3,77 \\
Pele & 27 & 3,51 \\
Sarcoma & 27 & 3,51 \\
Fígado & 18 & 2,34 \\
Ovário & 17 & 2,21 \\
Pâncreas & 15 & 1,95 \\
Vias Biliares & 15 & 1,95 \\
Linfoma & 8 & 1,04 \\
Rim & 8 & 1,04 \\
Bexiga & 7 & 0,91 \\
Cérebro & 7 & 0,91 \\
Endométrio & 4 & 0,52 \\
Canal Anal & 3 & 0,39 \\
Vulva & 3 & 0,39 \\
Leucemia & 2 & 0,26 \\
Mieloma Múltiplo & 2 & 0,26 \\
Pênis & 2 & 0,26 \\
\hline
\end{tabular}

Fonte: Silva WP, et al., 2021.

Quanto ao nível de capacidade funcional dos pacientes utilizando-se a Escala de Zubrod (ECOG), verificou-se que $23,77 \%$ estavam classificados como Performance Status 3 e 27,27\% performance 4 (Figura 1).

Figura 1 - Distribuição do nível de Capacidade funcional através da Escala PS-ECOG.

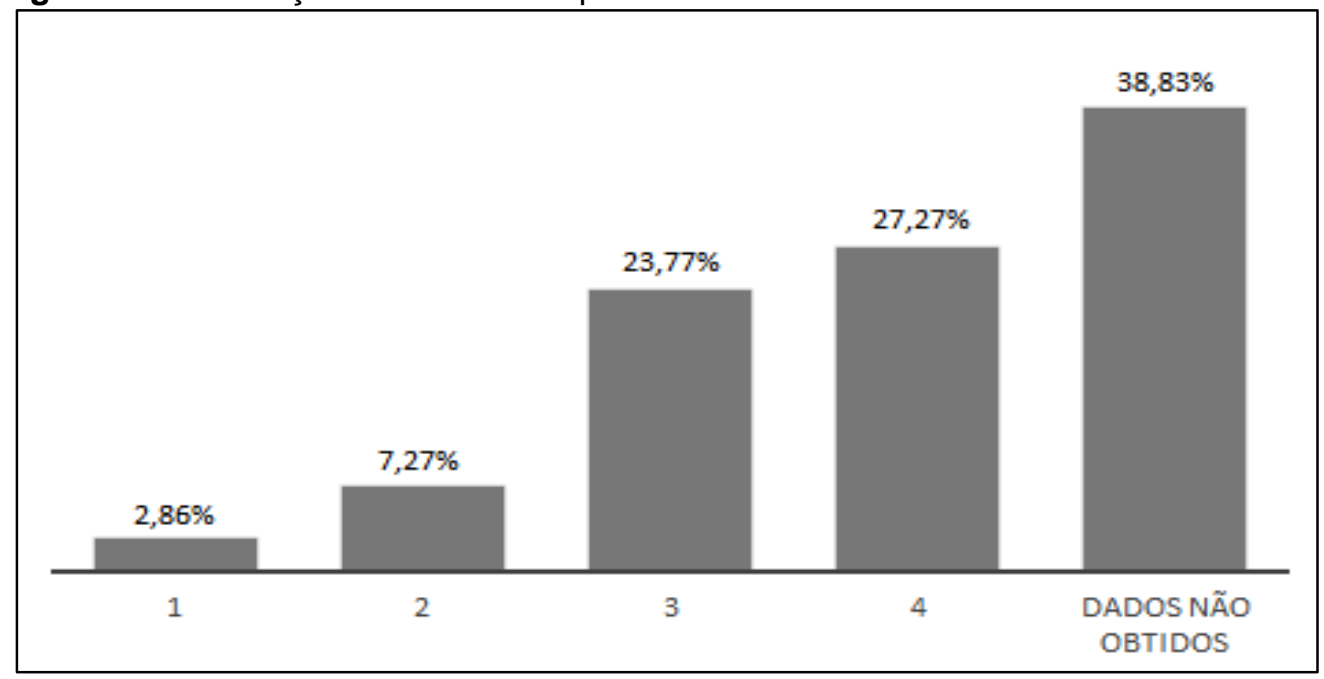

Fonte: Silva WP, et al., 2021. 
Os sintomas mais prevalentes durante a primeira internação foram $34,16 \%$ dor, $31,17 \%$ constipação e 28,05\% dispneia (Tabela 3).

Tabela 3 - Sintomas prevalentes nos pacientes atendidos no Serviço de Cuidados Paliativos.

\begin{tabular}{lcc}
\hline Sintomas & $\mathbf{N}$ & $\%$ \\
\hline Dor & 263 & 34,16 \\
Constipação & 240 & 31,17 \\
Dispneia & 216 & 28,05 \\
Edema de Membros Inferiores & 191 & 24,81 \\
Náuseas e Vômitos & 125 & 16,23 \\
Astenia & 110 & 14,29 \\
Hiporexia & 99 & 12,86 \\
Disfagia & 79 & 10,26 \\
Sangramento & 61 & 7,92 \\
Diarreia & 33 & 4,29 \\
\hline
\end{tabular}

Fonte: Silva WP, et al., 2021.

Quanto aos tratamentos aos quais os pacientes foram submetidos antes do ingresso no serviço de cuidados paliativos, verificou-se que $76,36 \%$ receberam algum tipo de tratamento modificador da doença e 23,64\% dos pacientes não foram submetidos a nenhum tipo de tratamento modificador antes de serem encaminhados ao serviço de Cuidados Paliativos. Daqueles que receberam algum tratamento oncológico anterior, 67,01\% fizeram Quimioterapia, 46,23\% Radioterapia, 19,87\% Cirurgia oncológica e 15,97\% Hormonioterapia. Após ingressarem no serviço de CP, 37,92\% foram atendidos na emergência da Unidade Hospitalar que mantém o serviço de Cuidados Paliativos; 44,03\% foram acompanhados ambulatorialmente e 84,94\% foram internados no serviço de CP (Tabela 4).

Tabela 4 - Tratamento modificador anterior e posterior à admissão no serviço de cuidados paliativos.

\begin{tabular}{lcc}
\hline Tratamento & N & $\%$ \\
\hline Tratamento anterior à admissão no serviço & & \\
\hline Quimioterapia & 516 & 67,01 \\
Radioterapia & 356 & 46,23 \\
Nenhum tratamento modificador & 182 & 23,64 \\
Cirurgia oncológica & 153 & 19,87 \\
$\quad$ Hormonioterapia & 123 & 15,97 \\
\hline Tratamento posterior à admissão no serviço & & \\
\hline Nenhum tratamento modificador & 710 & 92,21 \\
Radioterapia & 31 & 4,03 \\
Quimioterapia & 22 & 2,86 \\
Hormonioterapia & 4 & 0,52 \\
Cirurgia oncológica & 3 & 0,39 \\
\hline Atendimentos na emergência & & \\
\hline Não & 478 & 62,08 \\
Sim & 292 & 37,92 \\
\hline Atendimentos no ambulatório & & \\
\hline Não & 431 & 55,97 \\
Sim & 339 & 44,03 \\
\hline Internação hospitalar & & \\
\hline Sim & 654 & 84,94 \\
Não & 116 \\
\hline
\end{tabular}

Fonte: Silva WP, et al., 2021. 


\section{DISCUSSÃO}

Os pacientes foram encaminhados ao serviço de Cuidados Paliativos, pois não havia mais tratamento modificador da doença disponível, em função da evolução do cãncer. Nestes casos, os pacientes devem receber uma abordagem voltada para a qualidade de vida, sendo isto um alento para os pacientes e seus familiares (OLIVEIRA LC, 2019; FREIRE MEM, et al., 2018).

A existência desse centro de referência no Estado de Alagoas, certamente, não é suficiente para atender a demanda de pacientes com câncer avançado, conforme as recentes projeções estatísticas de incidência da doença. Sendo assim, pode-se inferir que uma parcela de pacientes oncológicos morre sem a assistência adequada, provavelmente num cenário de intenso sofrimento, o que gera importantes conflitos éticos, visto que o respeito ao princípio da dignidade humana e aos preceitos doutrinários do Sistema Único de Saúde precisam ser efetivos (INCA, 2019; VISENTIN A, et al., 2018; MENDES EC e VASCONCELLOS LCF, 2015; PILATTI P, et al., 2017).

O número reduzido de serviços de $\mathrm{CP}$ agrava a situação daqueles que precisam desse tipo de atendimento, que requer das equipes multidisciplinares e gestores de saúde pública investimentos para cuidar adequadamente dos portadores de padecimentos complexos e que não mais se beneficiam dos tratamentos curativos (FREIRE MEM, et al., 2018; MENDES EC e VASCONCELLOS LCF, 2015; ANCP, 2018).

Dos dados analisados, a distribuição dos pacientes em relação ao sexo demonstrou um maior percentual de indivíduos do sexo feminino na composição da amostra em comparação ao sexo masculino. Nesse aspecto, tal resultado se assemelha a outros achados em estudos que reforçaram a prevalência de mulheres com a doença (VISENTIN A, et al., 2018; FREIRE MEM, et al., 2018; SANTOS VNM, et al., 2020; SILVA IBS, et al., 2020; PEREIRA MME, et al., 2019). Historicamente, a mulher tende a procurar rotineiramente os serviços de saúde, com isso, o diagnóstico de câncer é facilitado e identificado mais precocemente, o que pode refletir no resultado encontrado nesta pesquisa, pois para terem acesso aos $\mathrm{CP}$ os doentes precisam ser referenciados e terem diagnóstico confirmado de câncer (BOTTON A, et al., 2017).

Foi possível constatar, também, que a maioria dos pacientes tinha idade acima de 56 anos e mais de $20 \%$ tinham entre 76 e 97 anos, ou seja, evidenciou-se o predomínio dos doentes paliativos na faixa etária mais avançada. Esse fato gera a reflexão sobre os cuidados com os pacientes idosos que, de maneira geral, já apresentam particularidades decorrentes das alterações fisiológicas inerentes ao processo de envelhecimento humano, portanto, tal contexto alcança maior complexidade haja vista o número de idosos com neoplasias que inexoravelmente os levarão à morte, o que por si só exige cuidados específicos ampliados que considerem as necessidades individuais desses enfermos (MIRANDA B, et al., 2016; BARBOSA KTF, et al., 2017; LEITE AKF e RIBEIRO KB, 2018).

Mais de $40 \%$ dos pacientes residiam fora de Maceió, resultado relevante, pois evidencia-se a necessidade de serem ofertados outros serviços de CP, sobretudo, no interior do Estado. Desses pacientes, alguns recebiam acompanhamento ambulatorial, o que exigia dos pacientes e seus cuidadores deslocamentos frequentes e regulares, fato que reforça a necessidade de se criar outros serviços para atender essa demanda. Além disso, em situações de intercorrências clínicas, o acesso ao serviço de emergência disponibilizado, provavelmente, era dificultoso. Desse modo, foi demonstrado a concentração da assistência na capital e a deficiência de suporte adequado no interior do Estado, obrigando aos pacientes deixarem seus locais de origem para serem assistidos na capital. Caracterização demográfica semelhante foi observada no estudo de Freire MEM, et al. (2018) no Estado da Paraíba ao discorrer sobre a qualidade de vida de pacientes com câncer que buscavam acesso adequado à saúde em João Pessoa, capital do Estado.

Em relação ao fluxo da assistência oncológica em Alagoas e o encaminhamento dos pacientes ao serviço de Cuidados Paliativos, a maioria foi encaminhada pelo Centro de Oncologia do próprio hospital que mantém o serviço de Cuidados Paliativos. Esse resultado demonstra que o hospital adota uma política de atendimento integral aos pacientes com câncer, a partir da confirmação do diagnóstico, e oferece tratamentos cirúrgicos, quimioterápico e radioterápico e, quando necessário, cuidados paliativos. Assegurando, assim, os direitos garantidos na política de atenção oncológica e na regulamentação dos CACONs no Brasil (BRASIL, 2013). 
Os sítios primários identificados como os mais prevalentes convergem com a literatura, sendo que a maioria dos pacientes foi acometida com câncer de cabeça e pescoço, seguidamente pelo câncer de pulmão. Este resultado evidencia a necessidade de adequação das políticas de rastreamento populacional e de educação em saúde, pois aponta para uma descoberta tardia da doença e a manutenção de hábitos que são prevalentes no desenvolvimento desse tipo de tumor como tabagismo, e com isso, mais pacientes evoluirão para estágios avançados e, muitas das vezes, sem tratamento modificador disponível, logo precisarão de CP (INCA, 2019; LEITE AKF e RIBEIRO KB, 2018).

Também é necessário, maiores reflexões sobre os achados referentes aos tumores ginecológicos, colo de útero e mama, pois são indicativos de que as políticas de prevenção e rastreio não têm sem sido eficazes, corroborando para esta análise, segundo o Inca (2019) as projeções dos números de casos novos desses tumores apontam para a ocorrência de 66.280 novos casos de câncer de mama e 16.950 de colo de útero. Além disso, esse resultado apresenta relação direta com o sexo mais prevalente no serviço, mulheres. Outro fator importante é o câncer de colo de útero ser o terceiro mais prevalente, seguido de câncer de mama. A pobreza, a baixa escolaridade, a falta de informação, o rastreamento e a prevenção ineficazes, provavelmente, são responsáveis pelo resultado (INCA, 2019; MASCARENHAS MS, et al., 2020; MEDEIROS GC, et al., 2020; BARBOSA IR, et al., 2016; MEDEIROS GC, et al., 2015).

Quanto ao declínio do nível de capacidade funcional dos pacientes recém admitidos no serviço, verificado pela Escala de Zubrod (ECOG), a maioria dos pacientes apresentou PS (ECOG) 4. Essa escala classifica a funcionalidade dos pacientes, onde o paciente no nível 4 se encontra acamado. Esse resultado pode ser justificado como um indicativo do avanço da doença que evoluiu de tal forma que prejudica a funcionalidade dos pacientes e interfere, evidentemente, na qualidade de vida (OKEN MM, et al.; 1982; PEREIRA EEB, et al., 2014).

Dentre os sintomas refratários, a dor foi o mais presente, exigindo dos profissionais o manejo dessa manifestação clínica. Para tanto, foram administrados esquemas de analgesia com opióides, os quais estão associados à constipação, segundo sintoma mais prevalente nessa pesquisa (LIMA MB e PEREIRA MCA, 2017). A dispneia, que é mais frequente nos pacientes com câncer de pulmão ou metástase pulmonar, representou $28,05 \%$ dos pacientes, esse resultado assemelha-se à literatura, que também indica a prevalência desse sintoma nos pacientes com câncer avançado (FREIRE MEM, et al., 2018).

A agressividade do câncer gera conflitos éticos uma vez que as manifestações e intercorrências clínicas apresentadas causam desconfortos ao paciente. Com isso, cada vez mais é preciso uma formação ampla que possibilite a aquisição de competências e habilidades no contexto paliativo, caso contrário, o doente que sofre, continuará sofrendo indubitavelmente (PILATTI P, et al., 2017; SANTOS VNM, et al., 2020; SILVA IBS, et al., 2020; SANTOS RB, et al., 2019). Com isso, muitas considerações podem ser feitas na tentativa de se obter uma compreensão acerca do contexto brasileiro sobre a atenção em CP, o que evidentemente precisa incluir uma análise da formação de profissionais da área de saúde, a qual se evidencia deficiente em muitos aspectos, não propondo aos futuros profissionais da saúde uma preparação que contemple temáticas em CP na atenção aos pacientes (LEMOS CFP, et al., 2017; FREITAS ED, 2017).

Com o avanço da doença, os tratamentos modificadores não se mostraram mais eficazes, portanto, podem ser considerados tratamentos fúteis, desproporcionais, isto é, não mais teriam os resultados esperados e provocariam sofrimento. Sendo assim, o princípio da proporcionalidade terapêutica deve prevalecer e, nesta pesquisa a maioria dos pacientes não foi submetida a nenhum tratamento com fins curativos (MARTA GN, et al., 2010). Ressalta-se, também, que foi possível compreender que a assistência oncológica na perspectiva paliativista esteve sempre à disposição dos pacientes, procurando mantê-los o mais confortável possível nos seus últimos dias de vida. Desse modo, os pacientes tinham acesso à emergência oncológica, ao ambulatório e quando apresentavam condições de difícil controle eram internados, por conseguinte, a maioria foi a óbito no próprio serviço de CP.

Verificou-se, também, que uma parcela dos pacientes foi atendida na emergência do serviço de Cuidados Paliativos, fato esse que reforça a complexidade dos quadros apresentados pelos pacientes com câncer. 
Além disso, é possível inferir que a busca desse tipo de assistência relaciona-se à gravidade e ao avanço da doença, como demonstrado em um estudo com pacientes oncológicos atendidos num centro de emergência, que mostrou que 70,7\% tinham doença em estágios avançados (MIRANDA B, et al., 2016).

Conforme foi demonstrado na coleta de dados do presente estudo, $23,64 \%$ dos prontuários dos pacientes eram de pessoas virgens de terapias modificadoras, com isso, representam uma parcela de doentes que descobrem o câncer mais tardiamente, com a doença em estado avançado e que encontram nos cuidados paliativos o suporte necessário ante à sua condição de saúde. Evidentemente, essa não é uma realidade restrita ao serviço em que esse trabalho foi realizado, pois situação semelhante foi identificada no trabalho de Miranda B, et al. (2016), no qual uma quantidade significativa dos pacientes também não foi submetida a tratamentos modificadores do curso da doença. Sendo assim, esse estudo levanta dados importantes que apontam para a ineficiência da atenção básica em trabalhar adequadamente a prevenção e o diagnóstico do câncer numa fase curativa.

Diante desses resultados, fica evidente que é necessário haver reflexões sobre a maneira como os pacientes são cuidados, sobretudo em contextos onde a busca pela cura do câncer não se apresenta mais viável. Neste caso, a obrigação moral e ética para com esses doentes gera o dever de se ampliar a discussão inerente a essa temática e discutir amplamente as questões de fim de vida.

Não obstante, estudos que proporcionem estatísticas que permitam aos poucos serviços existentes conhecerem as necessidades dos pacientes em condição paliativa e com câncer avançado e com isso fundamentarem melhorias ainda não são tão frequentes, sendo úteis maiores investigações para esse fim, o que pode gerar estímulo para a expansão desses centros à medida que se se adquire melhores percepções sobre a importância de se ter bons serviços de atenção aos pacientes portadores de neoplasias, afim de amparar essa parcela de pacientes. Como contribuição, este estudo também aponta para a eficácia da assistência ambulatorial e hospitalar fornecida pelos serviços de CP atuantes no Brasil e acrescenta-se a isso a possibilidade de gerar subsídios que norteiem a implantação de novos serviços desse tipo de assistência.

\section{CONCLUSÃO}

Diante dos resultados encontrados foi possível concluir que os pacientes que recorrem à assistência paliativa no Estado de Alagoas são predominantemente mulheres, de faixas etárias mais avançadas, residentes na capital do Estado, mas com uma parcela expressiva proveniente do interior, com comprometimento funcional e que necessitam de manejo apropriado dos sintomas, sendo encaminhados por um serviço especializado de oncologia da cidade de Maceió quando não apresentam respostas aos tratamentos curativos da doença. Foi possível constatar, também, que os tumores de cabeça e pescoço, pulmão e ginecológicos foram os mais prevalentes e que, em função das condições que esses pacientes apresentavam, os levavam a buscar os serviços de emergência e ambulatorial para controle de sintomas. Em vista disso, é justo afirmar que se faz necessário expandir serviços de Cuidados Paliativos, com a disponibilização de mais leitos, contando com profissionais qualificados.

\section{FINANCIAMENTO}

Fundação de Amparo à Pesquisa do Estado de Alagoas. Bolsa de Iniciação Científica. Processo: 60030 000819/2017 (16). Direcionado a Weslley Paixão da Silva.

\section{REFERÊNCIAS}

1. ACADEMIA NACIONAL DE CUIDADOS PALIATIVOS. Análise situacional e recomendações para estruturação de programas de cuidados paliativos no brasil. 2018. Disponível em: https://paliativo.org.br/analise-situacionalrecomendacoes-ancp-desenvolvimento-cuidados-paliativos-brasil. Acessado em: 01 de maio de 2021.

2. BARBOSA IR, et al. Desigualdades regionais na mortalidade por câncer de colo de útero no Brasil: tendências e projeções até o ano 2030. Ciênc. saúde coletiva, 2016; 21(1): 253-262.

3. BARBOSA KTF, et al. Envelhecimento e vulnerabilidade individual: um panorama dos idosos vinculados à estratégia saúde da família. Texto contexto - enferm, 2017; 26(2): e2700015. 
4. BOTTON A, et al. Diferenças de gênero no acesso aos serviços de saúde: problematizações necessárias. Mudanças Psicol Saúde, 2017; 25(1): 67-72.

5. BRASIL. Lei n. 12.732 de 22 de novembro de 2012. Dispõe sobre o primeiro tratamento de paciente com neoplasia maligna comprovada e estabelece prazo para seu início. 2012. Disponível em: http://www.planalto.gov.br/ccivil_03/_ato2011-2014/2012/lei//12732.htm. Acessado em: 06 de março de 2021

6. BRASIL. Portaria M/SAS no 874 de 16 de maio de 2013. Política Nacional para a Prevenção e Controle do Câncer na Rede de Atenção à Saúde das Pessoas com Doenças Crônicas no âmbito do Sistema Único de Saúde (SUS). 2013. Disponível em: http://bvsms.saude.gov.br/bvs/saudelegis/gm/2013/prt0874_16_05_2013.html. Acessado em: 06 de marco de 2021.

7. FREIRE MEM, et al. Qualidade de vida relacionada à saúde de pacientes com câncer em cuidados paliativos. Texto contexto - enferm, 2018; 27(2): e5420016.

8. FREITAS ED. Manifesto pelos cuidados paliativos na graduação em medicina: estudo dirigido da Carta de Praga. Rev. Bioét, 2017; 25(3): 527-535.

9. FURTADO MEMF e LEITE DMC. Cuidados paliativos sob a ótica de familiares de pacientes com neoplasia de pulmão. Interface (Botucatu), 2017; 21(63): 969-980.

10. HAUN MW, et al. Early palliative care for adults with advanced cancer. Cochrane Database of Systematic Reviews, 2017;6(6):CD011129.

11. INCA - INSTITUTO NACIONAL DE CÂNCER JOSÉ ALENCAR GOMES DA SILVA. Estimativa 2020: incidência de câncer no Brasil /Coordenação de Prevenção e Vigilância. 2019. Disponível em: https://www.inca.gov.br/publicacoes/livros/estimativa-2020-incidencia-de-cancer-no-brasil. Acessado em: 05 de abril de 2021.

12. KAVALIERATOS D, et al. Association Between Palliative Care and Patient and Caregiver Outcomes A Systematic Review and Metaanalysis. JAMA, 2016; 316(20): 2104-2114.

13. LEITE AKF, RIBEIRO KB. Older adults with cancer in the city of São Paulo: what factors determine the place of death?.Rev. Saúde Pública, 2018; 52: 66.

14. LEMOS CFP, et al. Avaliação do Conhecimento em Cuidados Paliativos em Estudantes durante o Curso de Medicina. Rev. bras. educ. med, 2017; 41(2): 278-282.

15. LIMA MB, PEREIRA MCA. Constipation in patients treated with opioids: an integrative review. Rev Bras Promoç Saúde, 2017;30(2): 275-282.

16. MARTA GN, et al. Cuidados paliativos e ortotanásia. Diagn. Trat, 2010; 5(2): 58-60.

17. MASCARENHAS MS, et al. Conhecimentos e Práticas de Usuárias da Atenção Primária à Saúde sobre o Controle do Câncer do Colo do Útero. Revista Brasileira de Cancerologia, 2020; 66(3): e-01030.

18. MEDEIROS GC, et al. Análise dos determinantes que influenciam o tempo para o início do tratamento de mulheres com câncer de mama no Brasil. Cad. Saúde Pública, 2015; 31(6): 1269-1282.

19. MEDEIROS GC, et al. Fatores Associados ao Atraso entre o Diagnóstico e o Início do Tratamento de Câncer de Mama: um Estudo de Coorte com 204.130 Casos no Brasil. Revista Brasileira de Cancerologia, 2020; 66(3): e-09979.

20. MENDES EC, VASCONCELLOS LCF. Cuidados paliativos no câncer e os princípios doutrinários no SUS. Saúde Debate, 2015;39(106):881-92.

21. MIRANDA B, et al. Cancer patients, emergencies service and provision of palliative care. Rev. Assoc. Med. Bras, 2016; 62(3): 207-211.

22. OKEN MM, et al.Toxicity and response criteria of the Eastern cooperative oncology group. Am J Clin Oncol, 1982; Dec;5(6):649-55.

23. OLIVEIRA LC. Cuidados Paliativos: Por que Precisamos Falar sobre isso?. Revista Brasileira de Cancerologia, 2019; 65(4): e-04558.

24. OLIVEIRA MBP, et al. Atendimento domiciliar oncológico: percepção de familiares/cuidadores sobre cuidados paliativos. Esc. Anna Nery, 2017; 21(2): e20170030.

25. OMS. Organização Mundial da Saúde. Definition of Palliative Care. 2a edition. Geneva: WHO; 2002.

26. PEREIRA EEB, et al. Avaliação da capacidade funcional do paciente oncogeriátrico hospitalizado. Rev Pan-Amaz Saude, 2014; 5(4): 37-44.

27. PEREIRA MME, et al. Ângulo de Fase e Estado Nutricional em Indivíduos com Câncer Avançado em Cuidados Paliativos. Revista Brasileira de Cancerologia, 2019; 65(1): e-02272.

28. PILATTI $P$, et al. Cuidados paliativos oncológicos em um serviço público de atenção domiciliar. Rev Bras Med Fam Comunidade, 2017; 12(39):1-10.

29. RIBEIRO JR, POLES K. Cuidados Paliativos: Prática dos Médicos da Estratégia Saúde da Família. Rev. bras. educ. med, 2019; 43(3): 62-72.

30. SANTOS RB, et al. Estudo Observacional Retrospectivo sobre o Perfil de pacientes que Receberam Terapia de Sedação Paliativa em Unidade de Cuidados Paliativos de Hospital de Câncer no Brasil. Revista Brasileira de Cancerologia, 2019; 65(1): e-09324.

31. SANTOS VNM, et al. Qualidade de Vida de Pacientes Oncológicos em Cuidados Paliativos Domiciliares e Desafios da Prática Médica diante da Finitude da Vida. Revista Brasileira de Cancerologia, 2020; 66(4): e-02423.

32. SILVA IBS, et al. Avaliação da Qualidade de Vida de Pacientes Oncológicos em Cuidados Paliativos. Revista Brasileira de Cancerologia, 2020; 66(3): e-121122.

33. VISENTIN A, et al. Palliative therapy in adults with cancer: a cross-sectional study. Rev. Bras. Enferm, 2018; 71(2): 252-258. 